Review Article

\title{
Review of Preclinical and Clinical Studies of Bone Marrow-Derived Cell Therapies for Intracerebral Hemorrhage
}

\author{
Paulo Henrique Rosado-de-Castro, ${ }^{1,2}$ \\ Felipe Gonçalves de Carvalho, ${ }^{3,4}$ Gabriel Rodriguez de Freitas, ${ }^{5}$ \\ Rosalia Mendez-Otero, ${ }^{6}$ and Pedro Moreno Pimentel-Coelho ${ }^{6}$ \\ ${ }^{1}$ Instituto de Ciências Biomédicas, Universidade Federal do Rio de Janeiro, 21941-902 Rio de Janeiro, RJ, Brazil \\ ${ }^{2}$ Departamento de Radiologia, Universidade Federal do Rio de Janeiro, 21941-902 Rio de Janeiro, RJ, Brazil \\ ${ }^{3}$ Departamento de Neurocirurgia, Universidade do Estado do Rio de Janeiro, 20551-030 Rio de Janeiro, RJ, Brazil \\ ${ }^{4}$ Instituto Estadual do Cérebro Paulo Niemeyer, 20231-092 Rio de Janeiro, RJ, Brazil \\ ${ }^{5}$ Instituto D'Or de Pesquisa e Ensino, 22281-100 Rio de Janeiro, RJ, Brazil \\ ${ }^{6}$ Instituto de Biofísica Carlos Chagas Filho, Universidade Federal do Rio de Janeiro, 21941-902 Rio de Janeiro, RJ, Brazil \\ Correspondence should be addressed to Pedro Moreno Pimentel-Coelho; pedrompc@biof.ufrj.br
}

Received 7 February 2016; Accepted 1 June 2016

Academic Editor: Eva Mezey

Copyright (C) 2016 Paulo Henrique Rosado-de-Castro et al. This is an open access article distributed under the Creative Commons Attribution License, which permits unrestricted use, distribution, and reproduction in any medium, provided the original work is properly cited.

\begin{abstract}
Stroke is the second leading cause of mortality worldwide, causing millions of deaths annually, and is also a major cause of disabilityadjusted life years. Hemorrhagic stroke accounts for approximately 10 to $27 \%$ of all cases and has a fatality rate of about $50 \%$ in the first 30 days, with limited treatment possibilities. In the past two decades, the therapeutic potential of bone marrow-derived cells (particularly mesenchymal stem cells and mononuclear cells) has been intensively investigated in preclinical models of different neurological diseases, including models of intracerebral hemorrhage and subarachnoid hemorrhage. More recently, clinical studies, most of them small, unblinded, and nonrandomized, have suggested that the therapy with bone marrow-derived cells is safe and feasible in patients with ischemic or hemorrhagic stroke. This review discusses the available evidence on the use of bone marrowderived cells to treat hemorrhagic strokes. Distinctive properties of animal studies are analyzed, including study design, cell dose, administration route, therapeutic time window, and possible mechanisms of action. Furthermore, clinical trials are also reviewed and discussed, with the objective of improving future studies in the field.
\end{abstract}

\section{Introduction}

Stroke is the second cause of death in the world, behind ischemic heart disease [1]. About 10 to $27 \%$ of strokes are hemorrhagic in presentation [2]. It has been estimated that in 2013 there were a total of 6.5 million deaths due to stroke (49\% from hemorrhagic strokes) [3] and that stroke was the second greatest cause of disability-adjusted life years (DALYs), causing 113 million DALYs (42\% due to hemorrhagic strokes) [3].

Intracerebral hemorrhage (ICH) can be classified as primary or secondary, depending on the cause of the hemorrhage. Primary ICH is caused by a spontaneous rupture of small arteries by chronic hypertension or amyloid angiopathy. In secondary ICH, the hemorrhage is caused, for example, by trauma, vasculopathies, coagulopathies, and tumors, among other causes [4].

Unlike the ischemic infarct, which often has an acute onset, ICH usually has a progressive onset. Symptoms vary according to the location and size of the hematoma [5]. In $40 \%$ of the cases, the patient will have intraventricular hemorrhage, which is associated with a worse outcome and with an increased risk of developing hydrocephalus [4]. Delayed deterioration is not uncommon and is usually due to rebleeding, edema, hydrocephalus, or seizures.

Many studies have been trying to elucidate the best treatment option for this catastrophic pathology since there are still many controversies regarding the management of patients with ICH. For this reason, every three years the 
American Heart Association and the American Stroke Association perform a review of the literature and elaborate an evidence-based guideline for the management of ICH [6].

Despite all efforts, the overall 30-day mortality rate of ICH patients can reach $40-50 \%$ [7]. Although a large number of randomized medical trials have been completed, they all failed to prove any benefit of different drugs or surgical interventions in patients with ICH $[8,9]$. Current treatment is based on the prevention of secondary brain injury, including rebleeding and secondary brain ischemia. General measures, such as the control of blood pressure, reduction of intracranial pressure (ICP), and prevention of infections, are important to prevent secondary brain injuries [7]. Indeed, the presence of secondary clinical complications, such as pneumonia, brain edema, cardiac complications, and sepsis, was correlated with a higher mortality in surgically and conservatively treated patients [10].

The general practice is to operate on young patients with large superficial lobar hematomas with mass effect and that are leading to an uncontrolled increase in ICP. In addition, posterior fossa hematomas are usually surgically evacuated, when they are larger than $3 \mathrm{~cm}$ in diameter and are causing a mass effect on the brainstem. In summary, each medical decision should be individualized based on patient's neurosurgical condition, size and location of hematoma, patient's age, and family wishes. Normally, ICHs are evacuated through a regular craniotomy, with or without the use of a microscope. New types of surgery have been studied and tried, including minimally invasive techniques with smaller incisions. However there is a lack of evidence that those new techniques are more efficient than the regular craniotomy $[6,11]$.

In this scenario, cell-based therapies represent a promising approach for the treatment of hemorrhagic stroke. Accumulating evidence suggests that different types of stem cells have the potential to induce or accelerate functional recovery in animal models of ICH and subarachnoid hemorrhage (SAH) [12-14]. As described by $\mathrm{Hu}$ et al. [12], mesenchymal stem cells (MSC) and neural stem cells were the most frequent cell types investigated in these studies. Neural stem cells are defined by their capacity to self-renew and give rise to neurons and glial cells. They can be derived from embryonic stem cells and induced pluripotent stem cells or can be isolated from fetal and adult neurogenic niches [15]. In contrast, MSC are plastic adherent stromal cells, characterized by their ability to self-renew and differentiate into osteocytes, chondrocytes, and adipocytes, but not into cells of nonmesodermal origin. MSC are present in the bone marrow and in numerous other fetal and adult tissues, including the umbilical cord, placenta, and adipose tissue, from which they can be isolated and expanded in culture $[16,17]$. Furthermore, MSC can be generated from embryonic stem cells and induced pluripotent stem cells $[18,19]$.

In view of the increasing use of bone marrow-derived MSC (BM-MSC) in clinical trials for ischemic and hemorrhagic strokes [20], the aim of this review is to discuss current evidence from preclinical and clinical studies that have investigated the therapeutic potential of BM-MSC in $\mathrm{ICH}$. Because the bone marrow contains other cell types with potential therapeutic value in ICH, including lymphocytes, monocytes, endothelial progenitor cells, and hematopoietic stem/progenitor cells, which compose the mononuclear cell fraction [21], studies that have used bone marrow mononuclear cells (BM-MNC) were included in the present review.

\section{Preclinical Studies}

We searched the PubMed/Medline database for original articles in English that have evaluated the therapeutic role of bone marrow-derived cells in animal models of ICH and SAH. We identified 18 articles in which BM-MSC were transplanted and 1 article in which BM-MNC were transplanted. The collagenase model of ICH was used by 11 studies while the remaining studies used the autologous blood model of ICH (6 studies) and SAH (2 studies) (Figure 1(a)). Almost all studies used rats, with the exception of two studies that used mice and monkeys (Figure 1(b)).

\subsection{Cell Type, Dose, Administration Route, and Time Window} for Therapy. In 14 of the 19 studies, rat BM-MSC were used while 4 other studies used human BM-MSC (Figure 2(a)). The remaining study used rat BM-MNC and it was the only study that injected autologous cells. All studies found beneficial effects of the treatment on structural and/or functional outcomes, as summarized in Table 1.

Most of the studies that we identified (12 of 19 or $63,17 \%$ ) have injected BM-MSC or BM-MNC within the first 24 hours after the injury (Figure 2(b)). This is consistent with the fact that these cells are expected to modulate early events in the pathophysiology of ICH and SAH, by counteracting neuronal cell death and limiting detrimental inflammatory responses [22-26]. For instance, in a series of 3 studies, Seyfried and colleagues evaluated the effects of human BM-MSC transplantation in a model of autologous blood injection into the rat striatum [27-29]. Their data showed that doses ranging from $1 \times 10^{6}$ to $8 \times 10^{6}$ of BM-MSC, intravenously injected 24 hours after $\mathrm{ICH}$, were equally effective in reducing tissue loss and improving the neurological function of treated animals $[27,29]$. In contrast, the lower dose of $0.5 \times 10^{6}$ cells was not capable of inducing such protective effect [29].

They have also tested the intra-arterial route of injection, by injecting $1 \times 10^{6}$ cells into the internal carotid artery. Interestingly, the beneficial effects of intra-arterially delivered BM-MSC were only observed when the animals were pretreated with an intravenous injection of mannitol. The authors proposed that mannitol facilitated the entry of BMMSC in the perihematomal region, through its effects on the blood-brain barrier [28].

Different results were obtained by Zhang et al. [30], who compared the effects of 3 routes of rat BM-MSC administration on the motor recovery of rats subjected to a model of collagenase injection into the striatum. In their study, while the intra-arterial and intracerebroventricular routes were able to improve the motor dysfunction (in the beam balance test), the intravenous delivery of BM-MSC showed no effect. Major differences between this work and the studies of Seyfried [27-29] were the source of BMMSC (rat versus human cells, resp.), the model of $\mathrm{ICH}$, and 


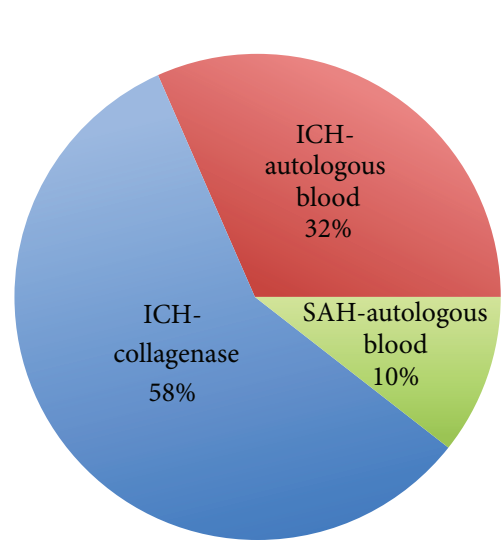

(a) Animal model

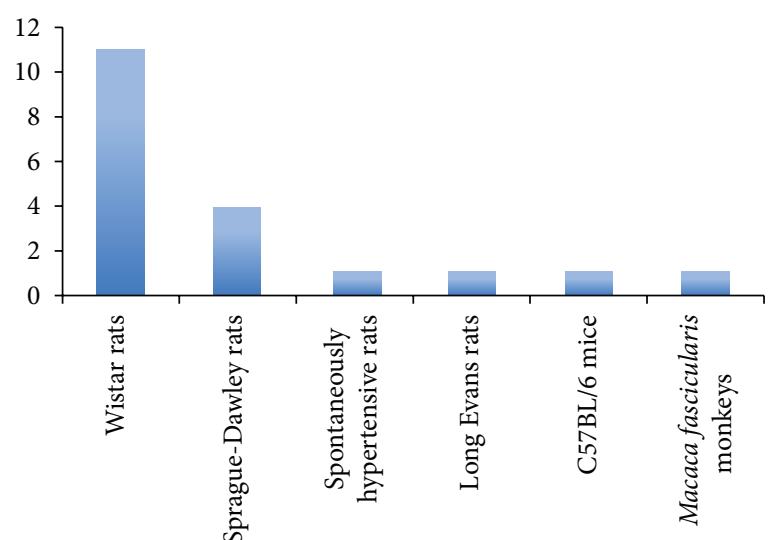

(b) Animal model (species)

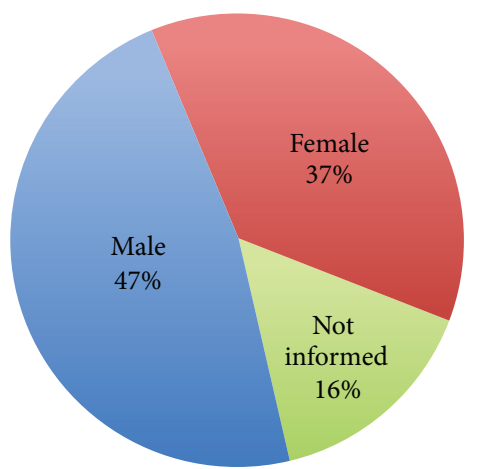

(c) Animal model (sex)

Figure 1: Charts summarizing the animal models (a), species (b), and sex of the animals (c) used in preclinical studies that have evaluated the potential of bone marrow-derived cells to treat hemorrhagic stroke.

the behavioral tests used, making it difficult to compare their results. However, all subsequent studies have shown that the intravenous administration of BM-MSC promotes neurological recovery in models of ICH $[23,25,31]$ and SAH [22].

The intracerebral route was the second most common route of BM-MSC administration in ICH models (Figure 2(c)) and also has led to an improvement of the functional outcome [24, 32-36]. Finally, Sun and coworkers [37] have shown that the intranasal administration could represent a less invasive option for the delivery of BM-MSC to the brain following ICH. Their work was based on previous studies showing that intranasally injected rat BM-MSC can migrate from the nasal mucosa through the cribriform plate into the intact and injured rodent brain $[38,39]$. In these studies, animals were pretreated with an intranasal injection of hyaluronidase 30 minutes before the application of cells, a protocol that facilitated the migration of BM-MSC to the brain [39].

Although the therapeutic window of BM-MSC administration for ICH and SAH has not been defined, there is evidence that the delayed treatment could still be effective. Vaquero and coworkers have shown that the IC administration of BM-MSC, 2 months after the induction of $\mathrm{ICH}$, improved the functional outcome of treated rats [34, 35]. This favorable effect on the neurological recovery was potentiated by embedding the cells in a platelet-rich plasma (PRP) scaffold. Notably, donor cells could be found near the injury site, up to 6 months after transplantation, and the use of PRP-derived scaffolds increased the number of engrafted cells. Although the delayed treatment did not affect the lesion volume, it stimulated the proliferation of endogenous cells in the subventricular zone (SVZ), one of the neurogenic niches of the adult brain [35]. Furthermore, one interesting study found that the neurological deficits were attenuated by BM-MSC, regardless of whether the cells were intracerebrally transplanted at 1 or 4 weeks after the induction of ICH in Macaca fascicularis monkeys. However, early treatment led to a better metabolic recovery, as assessed by serial positron emission tomography (PET) scans with ${ }^{18} \mathrm{~F}$ fluorodeoxyglucose $\left({ }^{18} \mathrm{~F}-\mathrm{FDG}\right)$ [32].

The majority of these studies have shown that BM-MSC and $\mathrm{BM}-\mathrm{MNC}$ migrated to the vicinity of the hemorrhagic lesion, regardless of the administration route. Several different techniques were employed to track the transplanted cells, but none of the studies have used noninvasive imaging techniques, such as bioluminescence imaging (BLI), fluorescence imaging (FLI), and radiopharmaceutical cell tracking (recently reviewed in [40]). The time point of the analysis varied among the studies, ranging from 6 hours to 6 months (Table 1), indicating that at least a fraction of the transplanted cells can survive for long periods in the brain. 


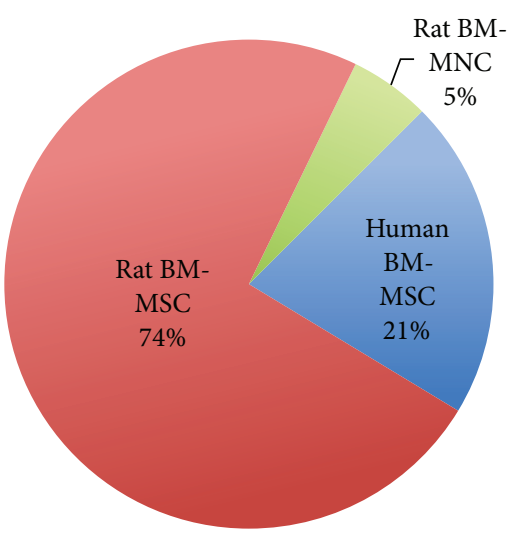

(a) Cell types

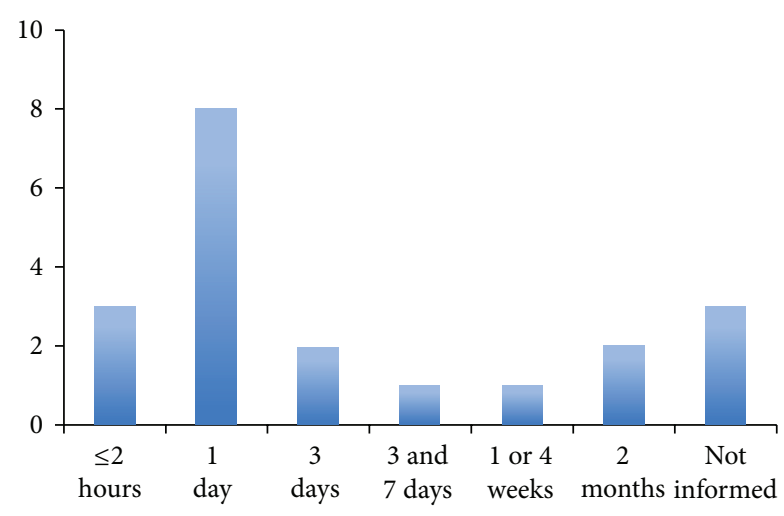

(b) Time of administration

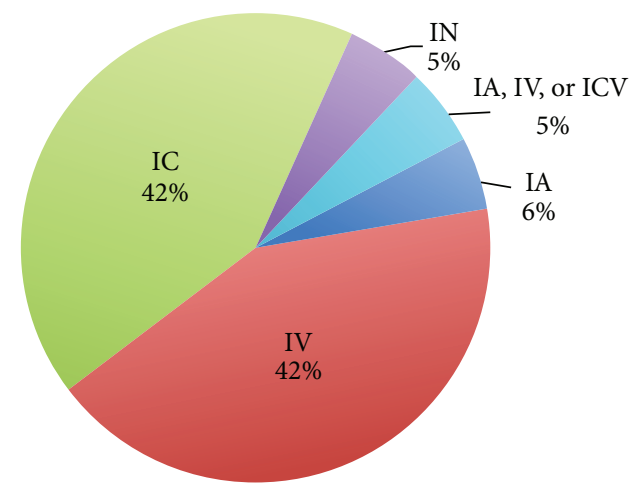

(c) Route of administration

FIGURE 2: Charts summarizing the type of bone marrow-derived cells (a), time of injection (b), and route of administration (c) in preclinical studies for hemorrhagic stroke.

2.2. Mechanisms of Action. Although it was initially hypothesized that BM-MSC and BM-MNC could differentiate into neural cells, this hypothesis has been challenged by many studies [17, 41-43]. Recent evidence supports the theory that the therapeutic action of bone marrow-derived cells is mediated by paracrine mechanisms, including the release of immune mediators, growth factors, and extracellular vesicles $[44,45]$. The success of BM-MSC-based therapies is founded on the ability of BM-MSC to sense changes in the local environment and modify their secretome in response to alarmins [46] and pathogen-associated molecular patterns [47]. Thus, a great research effort has been made to characterize the secretome of BM-MSC under different stimulatory conditions. For instance, Németh and colleagues [48] have demonstrated that lipopolysaccharide-stimulated BM-MSC release prostaglandin E2 which promotes the production of the anti-inflammatory cytokine IL-10 by macrophages. Similarly, by analyzing the secretome of BM-MSC cells with a new methodology, Milwid et al. [49] identified a previously unknown anti-inflammatory action of microfibrillarassociated protein 5 (MFAP5), which increased the production of IL-10 by lipopolysaccharide-stimulated monocytes and protected mice from a cytokine storm. Furthermore, extracellular vesicles, such as exosomes and microvesicles, are an important part of the BM-MSC secretome, as they can transfer functional mRNAs, miRNAs, proteins, and bioactive lipids with immunoregulatory and neuroprotective activities to target cells $[45,50,51]$.

The anti-inflammatory role of transplanted BM-MSC and BM-MNC was evidenced by their ability to decrease the number of microglial cells/macrophages and neutrophils in the perihematomal region and to attenuate the expression of proinflammatory cytokines in the brain and/or plasma in animal models of ICH [24-26]. The anti-inflammatory protein TNF- $\alpha$ stimulated gene/protein 6 (TSG-6) probably mediated at least part of these effects, as suggested by Chen et al. [25], who found increased levels of TSG-6 in the brain of rats treated with an intravenous injection of BM-MSC 2 hours after the induction of ICH. Previous studies have shown that a large fraction of intravenously injected BM-MSC is retained in the lungs [52] and that the pulmonary passage induces them to secrete high levels of TSG-6, which contributes to the healing of the infarcted myocardium [53]. In addition, it was recently observed that the intravenous infusion of BM-MSC-derived extracellular vesicles, containing $4 \mathrm{ng}$ of TSG-6, decreased the expression of the proinflammatory cytokine interleukin- $1 \beta$ in the brain and improved the cognitive function in a model of traumatic brain injury [54]. This is far lower than the dose of recombinant TSG-6 that promoted beneficial effects in this model of traumatic brain injury (50 $\mu \mathrm{g} / \mathrm{mouse}$, intravenous) [55], suggesting that there may be other molecules in BM-MSC-derived extracellular 


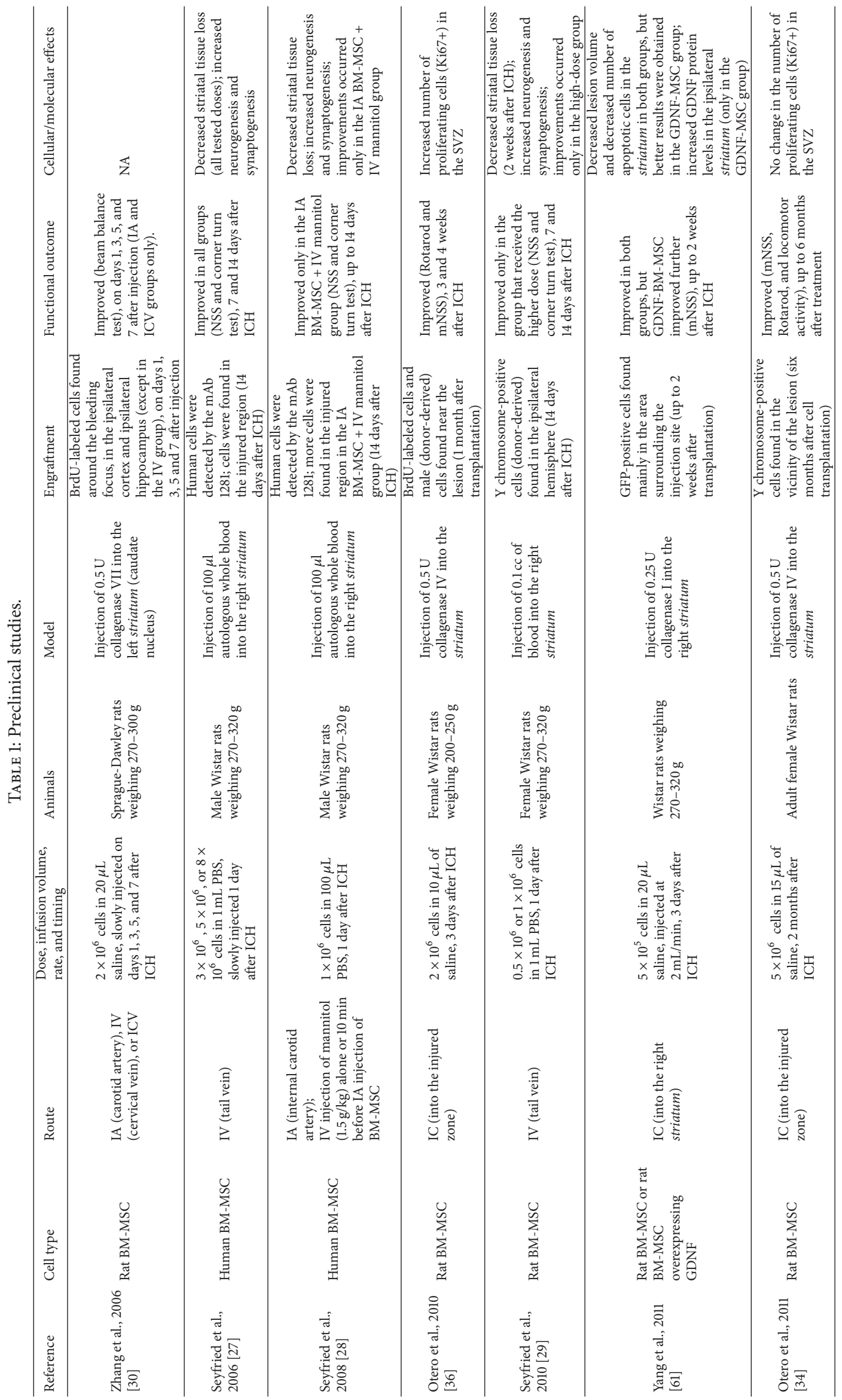




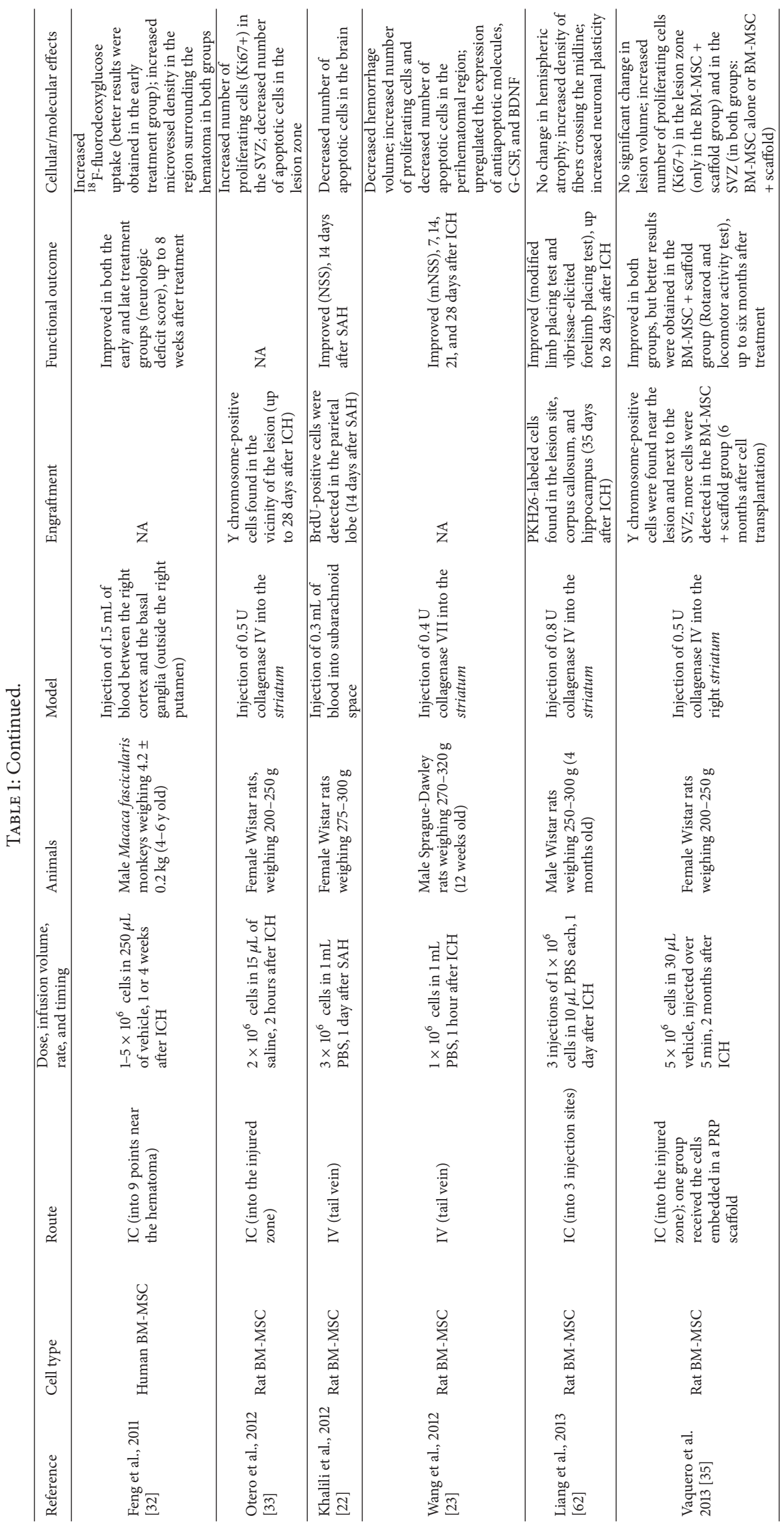




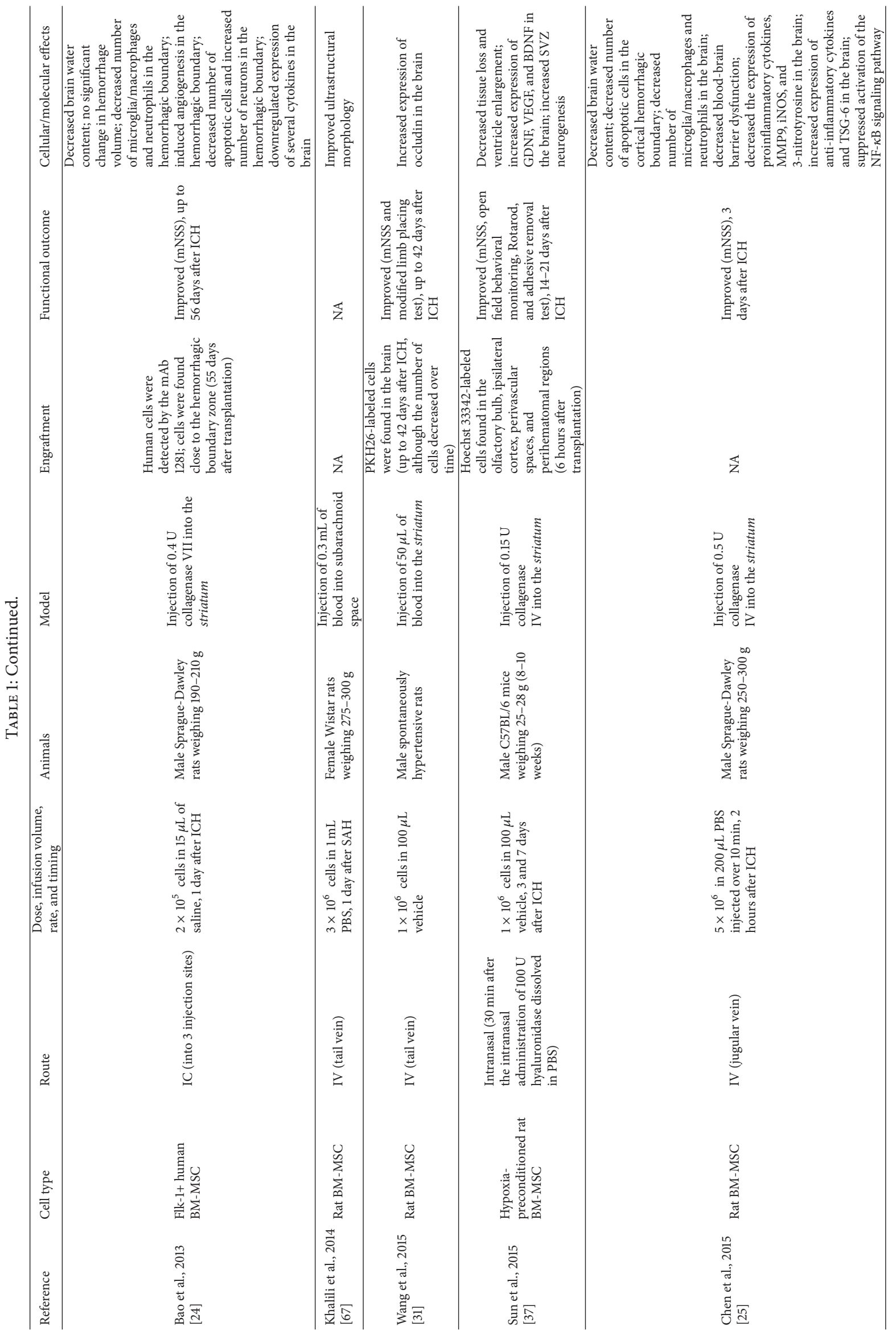




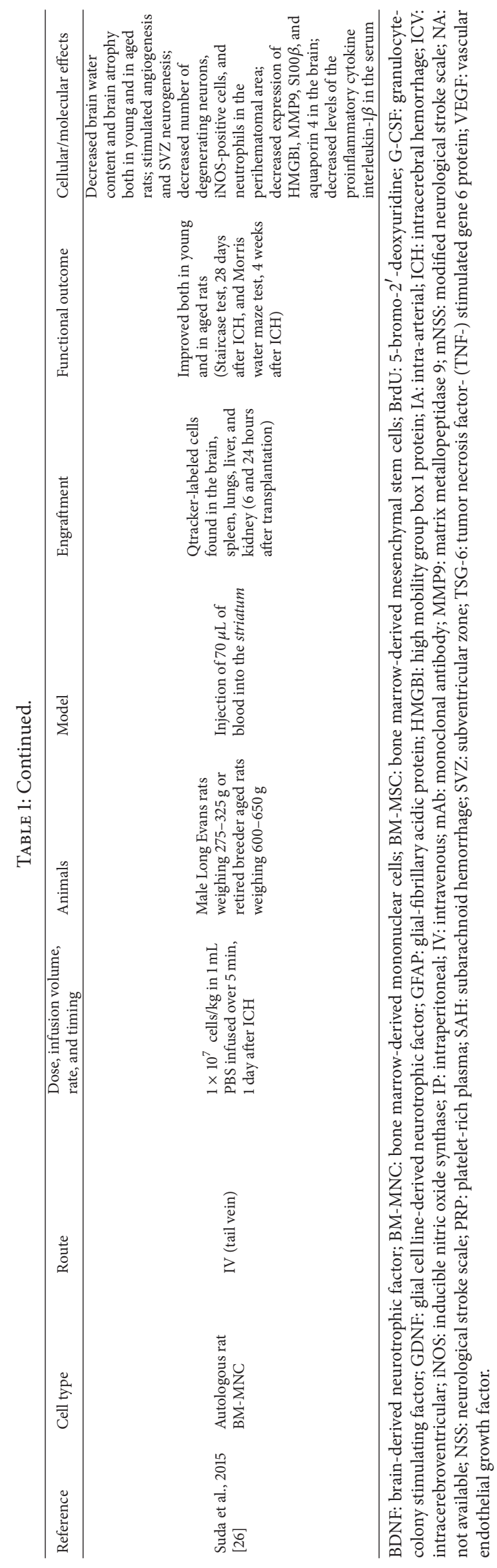


vesicles that act synergistically with TSG-6 or that there are differences in the bioavailability of TSG- 6 in these two conditions.

Studies using animal models of ischemic stroke have raised the possibility that umbilical cord blood- and bone marrow-derived cells could exert their anti-inflammatory action in the spleen $[56,57]$, where a large number of transplanted cells can be found for several days after transplantation [58-60]. Consistent with these findings, Suda et al. [26] observed that a significant number of intravenously administered BM-MNC migrated to the spleen in a rat model of ICH. However, it remains to be elucidated whether and how the interaction between transplanted cells and splenocytes contributes to the therapeutic effects of bone marrow-derived cells in this model.

Besides the anti-inflammatory action, BM-MSC and BMMNC have been shown to exert neuroprotection in models of $\mathrm{SAH}$ and ICH. Many studies have found a reduced number of apoptotic cells in the brain $[22-26,33,61]$ and/or a decrease in striatal tissue loss or lesion volume after the treatment [23, 27-29, 37, 61]. However, other studies showed no effects of the therapy on lesion volume $[24,35]$ or brain atrophy [62], despite the beneficial effect on functional recovery. A possible reason for this discrepancy is the fact that BMMSC and BM-MNC can stimulate mechanisms of brain plasticity [62] and regeneration, including synaptogenesis [27-29], angiogenesis [24, 26], and neurogenesis [33, 3537]. Moreover, the cell therapy can attenuate the blood-brain barrier dysfunction after ICH $[25,31]$. In this regard, it has been shown that the pulmonary passage may be an important step for the protection of the blood-brain barrier by intravenously delivered BM-MSC. Menge et al. [63] showed that the interaction between pulmonary endothelial cells and BM-MSC induced the latter to secrete tissue inhibitor of matrix metalloproteinase-3 (TIMP3), thereby increasing the serum levels of TIMP3 in a model of traumatic brain injury. Silencing the expression of TIMP3 in BM-MSC abrogated the protective effects of the cell therapy in the blood-brain barrier [63]. Further work from this group has revealed that TIMP3 also has a direct neuroprotective role both in vitro and in vivo [64].

2.3. Quality of Preclinical Studies. Two recent meta-analyses concluded that the quality of original articles investigating the efficacy of stem cell therapies in animal models of $\mathrm{ICH}$ is still low [12,13], as assessed by the Collaborative Approach to Meta-Analysis and Review of Animal Data from Experimental Stroke (CAMARADES) checklist [65]. For instance, studies using animals with relevant comorbidities (aged, hypertensive, or diabetic) are still rare. From the 19 studies included in our analysis, only 2 studies included animals with comorbidities. Wang et al. [31] subjected spontaneously hypertensive rats to the blood injection model of ICH and treated them with an intravenous injection of rat BM-MSC or vehicle. They showed that the cell therapy improved the recovery of behavioral function and increased the expression of the tight junction occludin in the blood-brain barrier of spontaneously hypertensive rats. However, the authors have not examined whether these effects were comparable to those reported in studies with normotensive rats. Another exception was the study of Suda and colleagues [26], who induced ICH in both young and aged rats. Animals received an intravenous injection of autologous BM-MNC or vehicle, 1 day after ICH. Both young and retired breeder aged rats benefited from the therapy, which decreased brain atrophy and edema and attenuated spatial learning deficits.

Other important parameters that are usually neglected or not informed by the authors are the control of body temperature during the surgeries, allocation concealment, blinded assessment of outcome, and sample size calculation $[12,13]$. In an elegant review, MacLellan and colleagues [66] identified and discussed some of the limitations of the current preclinical research on ICH. Among these limitations, they highlighted that despite the fact that behavioral data collected at acute time points might be confounded by other factors, such as the presence of brain edema, the functional outcome is rarely assessed at protracted time points. They also found that the neurological deficit score (NDS), which includes a battery of subtests, was the most used behavioral method in models of $\mathrm{ICH}$, whereas more sensitive tests were seldom performed. Indeed, among the 19 studies of ICH/SAH that we found, 13 had used NDS or modified versions of a neurological stroke scale (mNSS). Other sensorimotor behavioral tests that were used included the Rotarod (in 4 studies, 3 from the same group), the corner turn test (in 3 studies from the same group), the staircase test, and the adhesive removal test (used in 1 study each). Only one study also performed a cognitive test (Morris water maze task) [26] and two studies have not performed any behavioral assessments [33, 67]. We observed that the time of the last behavioral assessment varied widely, although almost all the studies have evaluated the animals for at least 2 weeks after the injury. In only one study, the behavioral analysis was restricted to the acute phase of stroke (Figure 3(a)) [25]. Similarly, the minimal number of animals per experimental group varied among the studies, ranging from 5 to 15 (Figure 3(b)).

Another criterion that is not included in the CAMARADES checklist is the inclusion of animals of both sexes with the separate analysis of data by sex, as recently recommended by the US National Institutes of Health (NIH) [68]. Among the 19 articles that we found, 7 studies have induced ICH only in females, 9 have subjected only male animals to $\mathrm{ICH}$, and the 3 remaining studies have not informed the sex of the experimental animals (Figure 1(c)). Although there is conflicting evidence regarding the existence of sexdependent differences in mortality and neurological outcome after $\mathrm{ICH}$, numerous preclinical studies have indicated that gonadal hormones affect the pathophysiology of ICH [69], which in turn could affect the response to a given therapy [70].

We also noticed that while the cell dose and the infusion volume were reported in all studies, the infusion velocity was not informed. Both the cell dose and the infusion velocity can influence the occurrence of adverse events, such as the formation of microocclusions and the development of stroke, after the intra-arterial administration of BM-MSC in rats $[71,72]$. These findings draw attention to the need for more studies on the long-term safety of cell therapies, such as 


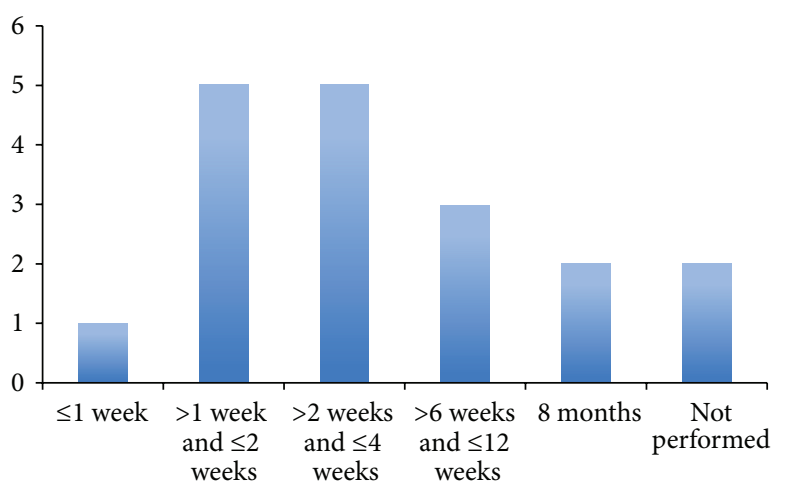

(a) Time of latest behavioral test

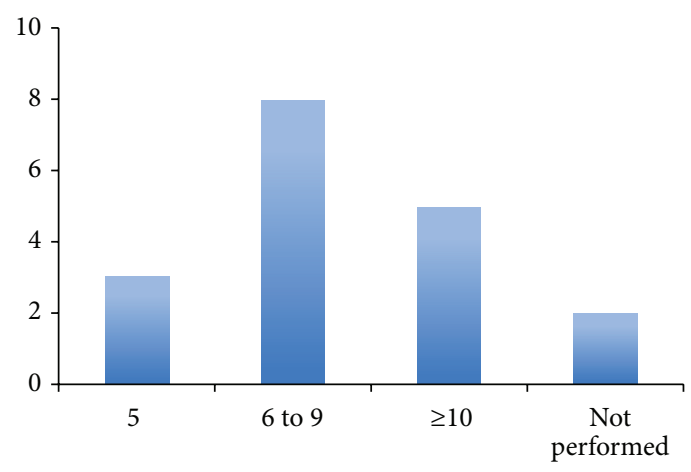

(b) Minimal number of animals per group (behavioral test)

FIGURE 3: Charts summarizing the timing of the latest behavioral test (a) and the minimal number of animals per experimental group (b) in preclinical studies that have evaluated the therapeutic potential of bone marrow-derived cells for hemorrhagic stroke.

the study by Donega et al. [73], who performed a systematic histopathological analysis of 38 organs, 14 months after the induction of neonatal hypoxia-ischemia and the intranasal transplantation of BM-MSC in mice. Their results showed no evidence of any adverse effects in the brain, nasal turbinates, or other peripheral organs.

\section{Clinical Studies}

We searched the PubMed/Medline database for original articles in English. We found 5 articles involving 5 different trials of cell therapies with a total of 188 treated patients with hemorrhagic stroke. Two trials were for basal ganglia hemorrhagic stroke and 3 for ischemic or hemorrhagic strokes (Table 2). One trial performed intravenous injections, 1 intrathecal injections, 2 intracerebral injections, and 1 intracerebral administration followed by intrathecal injections (Table 2).

Suarez-Monteagudo and coworkers [74] carried out a study with an IC injection of BM-MNC, which had 3 subjects with ischemic lesions in the cortex, striatum, or thalamus and 2 subjects with hemorrhagic lesions in the striatum or thalamus, between 3 and 8 years after the stroke. The patients with hemorrhagic lesions received a total of 1.7 $\times 10^{7}$ to $5.5 \times 10^{7}$ BM-MNC by stereotactic implantation along different tracts next to the injury. The authors reported that no significant adverse events occurred in the 12-month follow-up. The authors described functional improvements at 12 months, with a decline in motor deficits assessed by Ashworth's Scale for Spasticity and the Medical Research Council Scale; an increase in the National Institutes of Health Stroke Scale (NIHSS), Barthel index (BI), and Scandinavian Stroke Scale; and improved locomotion and equilibrium, assessed by the Tinetti scale. Nevertheless, the absence of a control group, the small study size, and the unblinded evaluation do not permit conclusions concerning efficacy.

$\mathrm{Li}$ and collaborators [75] included subjects in a nonrandomized, phase I, single-blind study of BM-MNC therapy. Sixty patients received an intraparenchymal cell injection 5 to 7 days following basal ganglia hemorrhage, and 40 subjects were included in the control group. The total number of injected cells varied from $2.5 \times 10^{8}$ to $2.3 \times 10^{9}$ cells. The authors reported that after 6 months the NIHSS scores in the cell therapy group were significantly lower in comparison to the control group $(p<0.01)$, whereas the BI scores were higher $(p<0.01)$. Furthermore, the authors described that neurological and functional improvements occurred in $86.7 \%$ of the patients in the cell therapy group versus $42.5 \%$ in the control group $(p=0.001)$.

Bhasin and colleagues [76] carried out an unblinded nonrandomized trial with 4 subjects with ischemic middle cerebral artery (MCA) lesions and 2 with hemorrhagic MCA lesions, as well as 6 control subjects. Autologous BM-MSC were intravenously injected between 8 and 12 months after the stroke. Patients were evaluated through the Medical Research Council scale for muscle strength, the modified Ashworth Scale for spasticity, the Fugl-Meyer (FM) scale for motor recovery, and the modified BI. The authors reported that no therapy-related adverse effects occurred in the 6-month follow-up. Even though the analysis indicated an advance on the FM scale and modified BI after 2 and 6 months, no statistically significant difference was seen when comparing the BM-MSC-treated and control groups.

Sharma et al. [77] included patients with ischemic or hemorrhagic stroke in a phase 1, nonrandomized, open-label study. The authors stated that 30 patients were included but 6 were lost to follow-up. Of the remaining 24 patients, 10 had hemorrhagic and 14 ischemic lesions. Patients were treated 4 to 144 months after the stroke (mean 40.5 months) and received an intrathecal injection of $10^{6}$ cells multiplied by the body weight. Follow-up varied from 6 to 54 months (mean 30 months). The authors reported that 12 patients had improvement in ambulation, 10 in hand functions, 6 in standing balance, 9 in walking balance, and 10 patients in functional status. Three patients agreed to undergo PET with ${ }^{18}$ F-FDG before and after cell therapy. The authors reported an increase in brain metabolism after cell therapy in these patients.

Zhu and collaborators [78] carried out a clinical trial where 215 patients with ICH were included. Following surgical drainage and decompressive craniotomy, 114 patients agreed to receive the cell therapy while the remaining 101 


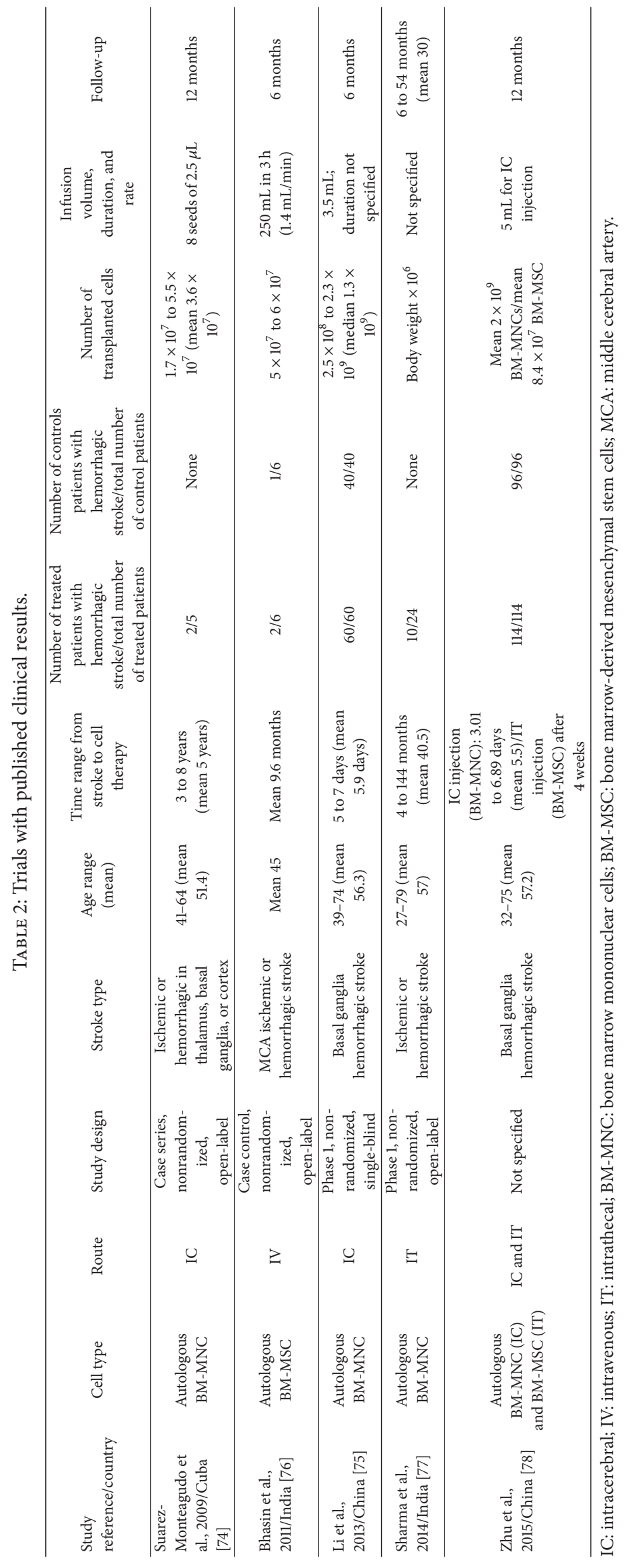


patients participated as a control group. Three to six days after the surgery, bone marrow harvest was performed and a mean of $4 \times 10^{9}$ BM-MNC was obtained. Half of the cell suspension was administered intracerebrally through indwelling drainage tubes that had been placed at the rim of the hematoma cavity. The other half of the cell suspension was separated for BM-MSC culture and a mean of $8.4 \times 10^{7}$ cells was injected after 4 weeks by the intrathecal route. Four patients from the cell therapy group and 5 patients from the control group were lost to follow-up. The authors reported that there was no significant difference in demographic variables including age, gender, neurological findings, mean lesion volumes, and surgical methods between both groups. The authors reported that NIHSS scores and Rankin scale in the cell therapy group were lower and Barthel scores were higher than in the control group at 6 and 12 months. The authors stated that no transplantation-related adverse events occurred in the follow-up.

3.1. Ongoing Clinical Studies. A search in clinicaltrials.gov showed 3 ongoing registered studies, which are estimated to enroll up to 280 subjects with ischemic or hemorrhagic strokes (Table 3). However, none of the studies informed the proportion of ischemic to hemorrhagic stroke patients that will be included, and it is therefore not possible to know how many hemorrhagic stroke patients will be included. The intrathecal injection of autologous BM-MNC was chosen by two of the trials and the intravenous injection of autologous BM-MSC was chosen by the remaining trial (Table 3 ).

\section{Discussion}

Several interrogations remain concerning the potential use of cell therapies for hemorrhagic stroke. Amongst the key issues to be resolved is defining the optimal cell type to be transplanted. In 2013 the US Food and Drug Administration released its "Guidance for Industry: Preclinical Assessment of Investigational Cellular and Gene Therapy Products" stating that whenever feasible, the tentative product that will be delivered to the patients should be evaluated in the preclinical studies [79]. However, the delivery of human cells in preclinical studies may be challenging due to immune responses of animals, which may lead to rejection of the cells [79].

There are marked differences between the predominant cell types that have been chosen by preclinical and clinical studies for ICH. Four out of 5 clinical studies used BMMNC while only 1 of the 19 preclinical studies studied this cell population. Moreover, while all clinical studies used autologous cells, either BM-MNC or BM-MSC, only 1 of the 19 preclinical studies used autologous cells. [26]. In the latter study, by Suda and collaborators, BM-MNC were collected 22 hours after ICH [26]. This approach was based on previous data demonstrating that the therapy with autologous BMMNC was more effective if the cells were harvested 22 hours after the ischemic event, compared to cells harvested 24 hours before MCA occlusion in rats [80]. Such results suggest that stroke may prime BM-MNC (or perhaps a subpopulation of BM-MNC) towards a protective phenotype.
Similar observations have been made in a model of acute intestinal infection with Toxoplasma gondii, where monocyte progenitors were primed for regulatory functions very early after infection, prior to their mobilization from bone marrow [81].

Notably, the transplantation of autologous poststroke BM-MNC is a feasible approach for the acute treatment of different types of stroke, since the BM-MNC fraction can be easily isolated in high yield and purity using density gradient media [20]. In contrast, the time required to expand BM-MSC in culture would not allow the transplantation of autologous BM-MSC in the acute phase of the injury, unless the cells had been banked for future use. Nevertheless, given their low immunogenicity, allogeneic MSC could be transplanted without the need for immunosuppressive drugs. This approach has been demonstrated in many preclinical and clinical studies for different diseases [20, 82]. For instance, Prochymal $^{\circledR}$, allogeneic culture-expanded adult human BMMSC, was the first stem cell product to receive conditional market authorization from Health Canada for the treatment of acute graft-versus-host disease in pediatric patients who are unresponsive to corticosteroid [83]. Different trials using culture-expanded allogeneic bone marrow-derived cells have also been designed for ischemic stroke, including from US companies Athersys, SanBio, and Stemedica, but their final results have not yet been reported $[84,85]$.

It is possible to manipulate BM-MSC before injection in an attempt to increase their survival, to induce a desired phenotype or to modify their migratory capacity. This can be done by changing culture conditions (e.g., three-dimensional culturing), by genetic manipulation, or by exposing the cells to growth factors, immunomodulators, or low doses of toxic factors, for example [86]. Wei and coworkers have demonstrated that BM-MSC upregulate the expression of the growth factors brain-derived neurotrophic factor (BDNF), glial cell line-derived neurotrophic factor (GDNF), and vascular endothelial growth factor (VEGF) after 48 hours of culture under hypoxic conditions [87]. They also have observed that the intranasal injection of hypoxia-preconditioned BMMSC (cultured for 24 hours under hypoxia) restored the expression of these same factors to near normal levels in the perihematomal region in mice [37].

Several lines of evidence have supported the notion that BM-MSC comprise a heterogeneous population of cells with distinct functions [88]. Therefore, the identification and enrichment of one or more subpopulations of BM-MSC with the desired functional attributes could represent a strategy to improve the therapeutic potential of BM-MSC. This was the approach used by Bao et al. [24], who investigated the effects of the intracerebral transplantation of fetal liver kinase- (Flk-) $1^{+}$human BM-MSC, a subpopulation of BMMSC that was the first stem cell product approved by the State Food and Drug Administration of China, in a rodent model of ICH. In sum, their results have shown that Flk-1 ${ }^{+}$ BM-MSC improved the neurological recovery through antiinflammatory, antiapoptotic, and proangiogenic effects.

Besides the intrapopulation heterogeneity, there may be differences in therapeutic efficacy among BM-MSC isolated from different donors. In an attempt to overcome this 


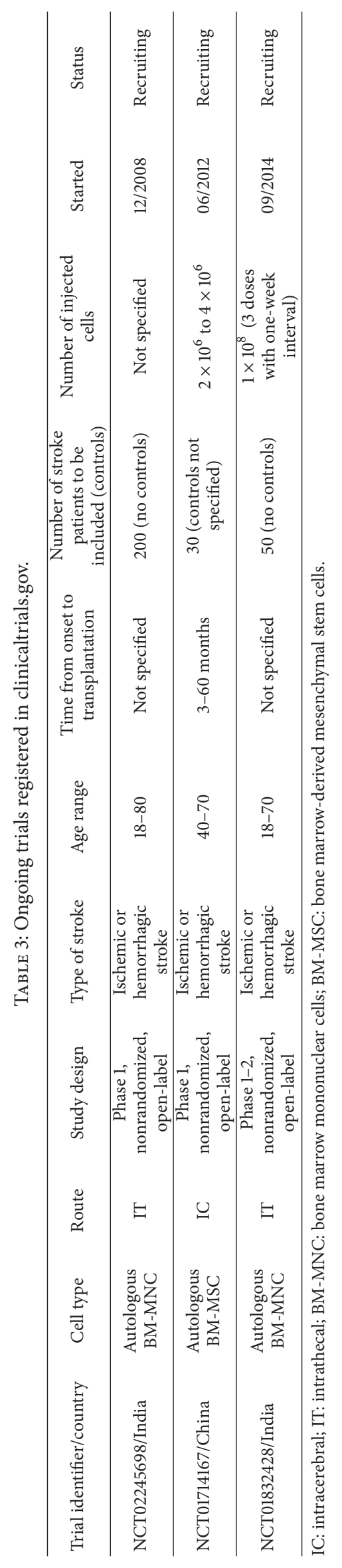


limitation, Prockop and colleagues have demonstrated that TSG-6 mRNA level could be used as a biomarker to predict the in vivo anti-inflammatory activity of human BM-MSC [89]. Accordingly, the establishment of potency assays is one of the main challenges for the development of cellular therapy products [90].

Finally, it has been demonstrated that MSC isolated from other tissues, such as the umbilical cord blood [91], umbilical cord tissue [92], or adipose tissue [93], can improve the behavioral recovery in animal models of ICH. However, given the intrinsic biological differences between MSC isolated from different tissues [94], it is still uncertain whether there are differences in their therapeutic potential.

The optimal cell dose also continues to be uncertain. The Stem Cell Therapies as an Emerging Paradigm in Stroke (STEPS) guidelines suggested dose conversion from preclinical studies based on body weight [95]. However, only one of the 5 clinical studies performed cell therapy based on body weight, and no preclinical study was used as rationale for dose conversion [77].

The use of imaging methods is another tool that can advance knowledge of numerous aspects, including cell migration and homing. Several methods can be employed to evaluate cell biodistribution. Although fluorescence and bioluminescence have been efficiently used to follow cells in animal studies for different neurological diseases, they have not been used in studies of cell therapies for hemorrhagic stroke and have limitations such as the restricted tissue penetration of light that does not allow their clinical use [96]. Superparamagnetic iron oxide nanoparticles (SPIOs), initially produced to identify hepatic tumors, may be used for exogenous cell labeling and tracking with MRI and has been used in a preclinical study with neural stem cell therapy for ICH [97]. However, SPIOs have restraints of exogenous contrasts, such as the chance of reduction with cell division. Furthermore, there are conflicting data on the impact of SPIOs in biological activities [98-101], and they have only been permitted for research studies.

For these reasons, nuclear medicine techniques such as PET and single photon emission computed tomography (SPECT) are important for the evaluation of cell migration and homing in vivo by indirect or direct assessments [40]. In indirect assessments, the uptake of ${ }^{18}$ F-FDG may be used to evaluate increased metabolism in the lesioned brain. One of the preclinical studies of BM-MSC therapy for $\mathrm{ICH}$ used ${ }^{18} \mathrm{~F}$ FDG and indicated improved metabolism up to 13 weeks after cell transplantation [32]. One of the clinical studies also used ${ }^{18} \mathrm{~F}-\mathrm{FDG}$ after intrathecal administration of autologous BMMNC in 3 of the 24 patients with ischemic or hemorrhagic stroke and reported increased brain metabolism one year after cell therapy [77]. However, the description of ${ }^{18} \mathrm{~F}$ FDG methods in the latter study was extremely limited and, therefore, does not allow any conclusive analysis. Moreover, the increase in ${ }^{18}$ F-FDG metabolism does not necessarily indicate the presence of transplanted cells or improved brain metabolism and may also occur if an inflammatory process takes place [102]. Cells may also be labeled with radiotracers such as Technetium-99m for direct cell tracking [40].
Although it has been proved feasible in preclinical and clinical studies for ischemic stroke [60, 103-108], it has not yet been employed in preclinical or clinical studies of cell therapies for hemorrhagic stroke.

\section{Conclusion}

Findings from preclinical reports indicate that cell transplantation may promote positive structural and functional effects in models of ICH and SAH. Nevertheless, the modes of action of these therapies are still under investigation. Clinical trials, most of them small, open-label, and nonrandomized, have been published and have reported that bone marrow-derived cell transplantation appears to be safe and feasible for hemorrhagic stroke. Nonetheless, there is a clear need for better preclinical and clinical studies, including randomized and blinded studies, to define the efficacy of cell transplantation for hemorrhagic stroke.

\section{Competing Interests}

The authors declare that there is no conflict of interests regarding the publication of this paper.

\section{Acknowledgments}

Dr. Paulo Henrique Rosado-de-Castro was supported by a grant (Universal 14/2014- 461701/2014-6) from Conselho Nacional de Desenvolvimento Científico e Tecnológico (CNPq). Dr. Pedro Moreno Pimentel-Coelho and Dr. Rosalia Mendez-Otero also wish to thank Fundação Carlos Chagas Filho de Amparo à Pesquisa do Estado do Rio de Janeiro (FAPERJ), Rede Nacional de Pesquisa em Acidente Vascular Cerebral-Departamento de Ciência e Tecnologia do Ministério da Saúde (DECIT), and Programa Ciência Sem Fronteiras-Coordenação de Aperfeiçoamento de Pessoal de Nível Superior (CAPES).

\section{References}

[1] R. Lozano, M. Naghavi, K. Foreman et al., "Global and regional mortality from 235 causes of death for 20 age groups in 1990 and 2010: a systematic analysis for the Global Burden of Disease Study 2010," The Lancet, vol. 380, no. 9859, pp. 2095-2128, 1990.

[2] V. L. Feigin, C. M. Lawes, D. A. Bennett, S. L. Barker-Collo, and V. Parag, "Worldwide stroke incidence and early case fatality reported in 56 population-based studies: a systematic review," The Lancet Neurology, vol. 8, no. 4, pp. 355-369, 2009.

[3] V. L. Feigin, R. V. Krishnamurthi, P. Parmar et al., "Update on the global burden of ischemic and hemorrhagic stroke in 19902013: the GBD 2013 study," Neuroepidemiology, vol. 45, no. 3, pp. 161-176, 2015.

[4] S. A. Mayer and F. Rincon, "Treatment of intracerebral haemorrhage," The Lancet Neurology, vol. 4, no. 10, pp. 662-672, 2005.

[5] N. Etminan, K. Beseoglu, and D. Hänggi, "Neurosurgery in hemorrhagic stroke," in PanVascular Medicine, P. Lanzer, Ed., pp. 1-24, Springer, Berlin, Germany, 2013.

[6] J. C. Hemphill III, S. M. Greenberg, C. S. Anderson et al., "Guidelines for the management of spontaneous intracerebral 
hemorrhage: a guideline for healthcare professionals from the American Heart Association/American Stroke Association," Stroke, vol. 46, no. 7, pp. 2032-2060, 2015.

[7] P. Fogarty Mack, "Intracranial haemorrhage: therapeutic interventions and anaesthetic management," British Journal of Anaesthesia, vol. 113, supplement 2, pp. ii17-ii25, 2014.

[8] D. Fiorella, S. L. Zuckerman, I. S. Khan, N. K. Ganesh, and J. Mocco, "Intracerebral hemorrhage: a common and devastating disease in need of better treatment," World Neurosurgery, vol. 84, no. 4, pp. 1136-1141, 2015.

[9] R. F. Keep, Y. Hua, and G. Xi, "Intracerebral haemorrhage: mechanisms of injury and therapeutic targets," The Lancet Neurology, vol. 11, no. 8, pp. 720-731, 2012.

[10] M. Stein, G. F. Hamann, B. Misselwitz, E. Uhl, M. Kolodziej, and M. H. Reinges, "In-hospital mortality and complication rates in surgically and conservatively treated patients with spontaneous intracerebral hemorrhage in central Europe: a populationbased study," World Neurosurgery, vol. 88, pp. 306-310, 2016.

[11] M. S. Greenberg, Handbook of Neurosurgery, Greenberg Graphics, Tampa, Fla, USA, 7th edition, 2010.

[12] Y. Hu, N. Liu, P. Zhang et al., "Preclinical studies of stem cell transplantation in intracerebral hemorrhage: a systemic review and meta-analysis," Molecular Neurobiology, 2015.

[13] X. Ma, J. Qin, B. Song et al., "Stem cell-based therapies for intracerebral hemorrhage in animal model: a meta-analysis," Neurological Sciences, vol. 36, no. 8, pp. 1311-1317, 2015.

[14] M. F. Cordeiro, "Stem cell therapy in intracerebral hemorrhage rat model," World Journal of Stem Cells, vol. 7, no. 3, pp. 618-629, 2015.

[15] F. H. Gage and S. Temple, "Neural stem cells: generating and regenerating the brain," Neuron, vol. 80, no. 3, pp. 588-601, 2013.

[16] M. Dominici, K. Le Blanc, I. Mueller et al., "Minimal criteria for defining multipotent mesenchymal stromal cells. The International Society for Cellular Therapy Position Statement," Cytotherapy, vol. 8, no. 4, pp. 315-317, 2006.

[17] C. M. Verfaillie and R. Abraham, "Neural differentiation and support of neuroregeneration of non-neural adult stem cells," Progress in Brain Research, vol. 201, pp. 17-34, 2012.

[18] K. Hynes, D. Menicanin, K. Mrozik, S. Gronthos, and P. M. Bartold, "Generation of functional mesenchymal stem cells from different induced pluripotent stem cell lines," Stem Cells and Development, vol. 23, no. 10, pp. 1084-1096, 2014.

[19] R. Gadkari, L. Zhao, T. Teklemariam, and B. M. Hantash, "Human embryonic stem cell derived-mesenchymal stem cells: an alternative mesenchymal stem cell source for regenerative medicine therapy," Regenerative Medicine, vol. 9, no. 4, pp. 453465, 2014.

[20] P. H. Rosado-de-Castro, P. M. Pimentel-Coelho, L. M. B. da Fonseca, G. R. de Freitas, and R. Mendez-Otero, "The rise of cell therapy trials for stroke: review of published and registered studies," Stem Cells and Development, vol. 22, no. 15, pp. 20952111, 2013.

[21] C. Pösel, K. Möller, W. Fröhlich et al., "Density gradient centrifugation compromises bone marrow mononuclear cell yield," PLoS ONE, vol. 7, no. 12, Article ID e50293, 2012.

[22] M. A. Khalili, M. Anvari, S. H. Hekmati-Moghadam, F. Sadeghian-Nodoushan, F. Fesahat, and S. M. Miresmaeili, "Therapeutic benefit of intravenous transplantation of mesenchymal stem cells after experimental subarachnoid hemorrhage in rats," Journal of Stroke and Cerebrovascular Diseases, vol. 21, no. 6, pp. 445-451, 2012.
[23] S.-P. Wang, Z.-H. Wang, D.-Y. Peng, S.-M. Li, H. Wang, and X.-H. Wang, "Therapeutic effect of mesenchymal stem cells in rats with intracerebral hemorrhage: reduced apoptosis and enhanced neuroprotection," Molecular Medicine Reports, vol. 6, no. 4, pp. 848-854, 2012.

[24] X.-J. Bao, F.-Y. Liu, S. Lu et al., "Transplantation of FLK-1+ human bone marrow-derived mesenchymal stem cells promotes behavioral recovery and anti-inflammatory and angiogenesis effects in an intracerebral hemorrhage rat model," International Journal of Molecular Medicine, vol. 31, no. 5, pp. 1087-1096, 2013.

[25] M. Chen, X. Li, X. Zhang et al., "The inhibitory effect of mesenchymal stem cell on blood-brain barrier disruption following intracerebral hemorrhage in rats: contribution of TSG6," Journal of Neuroinflammation, vol. 12, no. 1, article 61, 2015.

[26] S. Suda, B. Yang, K. Schaar et al., "Autologous bone marrow mononuclear cells exert broad effects on short- and long-term biological and functional outcomes in rodents with intracerebral hemorrhage," Stem Cells and Development, vol. 24, no. 23, pp. 2756-2766, 2015.

[27] D. Seyfried, J. Ding, Y. Han, Y. Li, J. Chen, and M. Chopp, "Effects of intravenous administration of human bone marrow stromal cells after intracerebral hemorrhage in rats," Journal of Neurosurgery, vol. 104, no. 2, pp. 313-318, 2006.

[28] D. M. Seyfried, Y. Han, D. Yang et al., "Mannitol enhances delivery of marrow stromal cells to the brain after experimental intracerebral hemorrhage," Brain Research, vol. 1224, pp. 12-19, 2008.

[29] D. M. Seyfried, Y. Han, D. Yang et al., "Localization of bone marrow stromal cells to the injury site after intracerebral hemorrhage in rats: laboratory investigation," Journal of Neurosurgery, vol. 112, no. 2, pp. 329-335, 2010.

[30] H. Zhang, Z. Huang, Y. Xu, and S. Zhang, "Differentiation and neurological benefit of the mesenchymal stem cells transplanted into the rat brain following intracerebral hemorrhage," Neurological Research, vol. 28, no. 1, pp. 104-112, 2006.

[31] C. Wang, Y. Fei, C. Xu, Y. Zhao, and Y. Pan, "Bone marrow mesenchymal stem cells ameliorate neurological deficits and blood-brain barrier dysfunction after intracerebral hemorrhage in spontaneously hypertensive rats," International Journal of Clinical and Experimental Pathology, vol. 8, no. 5, pp. 4715-4724, 2015.

[32] M. Feng, H. Zhu, Z. Zhu et al., "Serial 18F-FDG PET demonstrates benefit of human mesenchymal stem cells in treatment of intracerebral hematoma: a translational study in a primate model," Journal of Nuclear Medicine, vol. 52, no. 1, pp. 90-97, 2011.

[33] L. Otero, M. Zurita, C. Bonilla et al., "Allogeneic bone marrow stromal cell transplantation after cerebral hemorrhage achieves cell transdifferentiation and modulates endogenous neurogenesis," Cytotherapy, vol. 14, no. 1, pp. 34-44, 2012.

[34] L. Otero, M. Zurita, C. Bonilla et al., "Late transplantation of allogeneic bone marrow stromal cells improves neurologic deficits subsequent to intracerebral hemorrhage," Cytotherapy, vol. 13, no. 5, pp. 562-571, 2011.

[35] J. Vaquero, L. Otero, C. Bonilla et al., "Cell therapy with bone marrow stromal cells after intracerebral hemorrhage: impact of platelet-rich plasma scaffolds," Cytotherapy, vol.15, no. 1, pp. 3343, 2013. 
[36] L. Otero, C. Bonilla, C. Aguayo, M. Zurita, and J. Vaquero, "Intralesional administration of allogeneic bone marrow stromal cells reduces functional deficits after intracerebral hemorrhage," Histology and Histopathology, vol. 25, no. 4, pp. 453-461, 2010.

[37] J. Sun, Z. Z. A. Wei, X. Gu et al., "Intranasal delivery of hypoxiapreconditioned bone marrow-derived mesenchymal stem cells enhanced regenerative effects after intracerebral hemorrhagic stroke in mice," Experimental neurology, vol. 272, pp. 78-87, 2015.

[38] V. Donega, C. H. Nijboer, G. van Tilborg, R. M. Dijkhuizen, A. Kavelaars, and C. J. Heijnen, "Intranasally administered mesenchymal stem cells promote a regenerative niche for repair of neonatal ischemic brain injury," Experimental Neurology, vol. 261, pp. 53-64, 2014.

[39] L. Danielyan, R. Schäfer, A. von Ameln-Mayerhofer et al., "Intranasal delivery of cells to the brain," European Journal of Cell Biology, vol. 88, no. 6, pp. 315-324, 2009.

[40] P. H. Rosado-de-Castro, P. M. Pimentel-Coelho, B. Gutfilen et al., "Radiopharmaceutical stem cell tracking for neurological diseases," BioMed Research International, vol. 2014, Article ID 417091, 12 pages, 2014.

[41] M. Alvarez-Dolado, R. Pardal, J. M. Garcia-Verdugo et al., "Fusion of bone-marrow-derived cells with Purkinje neurons, cardiomyocytes and hepatocytes," Nature, vol. 425, no. 6961, pp. 968-973, 2003.

[42] R. F. Castro, K. A. Jackson, M. A. Goodell, C. S. Robertson, H. Liu, and H. D. Shine, "Failure of bone marrow cells to transdifferentiate into neural cells in vivo," Science, vol. 297, no. 5585, p. 1299, 2002.

[43] G. F. Barnabé, T. T. Schwindt, M. E. Calcagnotto et al., "Chemically-induced RAT mesenchymal stem cells adopt molecular properties of neuronal-like cells but do not have basic neuronal functional properties," PLoS ONE, vol. 4, no. 4, Article ID e5222, 2009.

[44] S. H. Ranganath, O. Levy, M. S. Inamdar, and J. M. Karp, "Harnessing the mesenchymal stem cell secretome for the treatment of cardiovascular disease," Cell Stem Cell, vol. 10, no. 3, pp. 244-258, 2012.

[45] S. Bruno, M. C. Deregibus, and G. Camussi, "The secretome of mesenchymal stromal cells: role of extracellular vesicles in immunomodulation," Immunology Letters, vol. 168, no. 2, pp. 154-158, 2015.

[46] E. Meng, Z. Guo, H. Wang et al., "High mobility group box 1 protein inhibits the proliferation of human mesenchymal stem cells and promotes their migration and differentiation along osteoblastic pathway," Stem Cells and Development, vol. 17, no. 4, pp. 805-813, 2008.

[47] R. S. Waterman, S. L. Tomchuck, S. L. Henkle, and A. M. Betancourt, "A new mesenchymal stem cell (MSC) paradigm: polarization into a pro-inflammatory $\mathrm{MSC1}$ or an immunosuppressive MSC2 phenotype," PLoS ONE, vol. 5, no. 4, Article ID e10088, 2010.

[48] K. Németh, A. Leelahavanichkul, P. S. T. Yuen et al., "Bone marrow stromal cells attenuate sepsis via prostaglandin E 2dependent reprogramming of host macrophages to increase their interleukin-10 production," Nature Medicine, vol. 15, no. 1, pp. 42-49, 2009.

[49] J. M. Milwid, J. S. Elman, M. Li et al., "Enriched protein screening of human bone marrow mesenchymal stromal cell secretions reveals MFAP5 and PENK as novel IL-10 modulators," Molecular Therapy, vol. 22, no. 5, pp. 999-1007, 2014.
[50] H. Xin, Y. Li, Y. Cui, J. J. Yang, Z. G. Zhang, and M. Chopp, “Systemic administration of exosomes released from mesenchymal stromal cells promote functional recovery and neurovascular plasticity after stroke in rats," Journal of Cerebral Blood Flow and Metabolism, vol. 33, no. 11, pp. 1711-1715, 2013.

[51] T. R. Doeppner, J. Herz, A. Görgens et al., "Extracellular vesicles improve post-stroke neuroregeneration and prevent postischemic immunosuppression," Stem Cells Translational Medicine, vol. 4, no. 10, pp. 1131-1143, 2015.

[52] U. M. Fischer, M. T. Harting, F. Jimenez et al., "Pulmonary passage is a major obstacle for intravenous stem cell delivery: the pulmonary first-pass effect," Stem Cells and Development, vol. 18, no. 5, pp. 683-691, 2009.

[53] R. H. Lee, A. A. Pulin, M. J. Seo et al., "Intravenous hMSCs improve myocardial infarction in mice because cells embolized in lung are activated to secrete the anti-inflammatory protein TSG-6," Cell Stem Cell, vol. 5, no. 1, pp. 54-63, 2009.

[54] D.-K. Kim, H. Nishida, S. Y. An, A. K. Shetty, T. J. Bartosh, and D. J. Prockop, "Chromatographically isolated CD63+CD81+ extracellular vesicles from mesenchymal stromal cells rescue cognitive impairments after TBI," Proceedings of the National Academy of Sciences of the United States of America, vol. 113, no. 1, pp. 170-175, 2016.

[55] J. Watanabe, A. K. Shetty, B. Hattiangady et al., "Administration of TSG-6 improves memory after traumatic brain injury in mice," Neurobiology of Disease, vol. 59, pp. 86-99, 2013.

[56] M. Vendrame, C. Gemma, K. R. Pennypacker et al., "Cord blood rescues stroke-induced changes in splenocyte phenotype and function," Experimental Neurology, vol. 199, no. 1, pp. 191-200, 2006.

[57] S. Schwarting, S. Litwak, W. Hao, M. Bähr, J. Weise, and H. Neumann, "Hematopoietic stem cells reduce postischemic inflammation and ameliorate ischemic brain injury," Stroke, vol. 39, no. 10, pp. 2867-2875, 2008.

[58] S. A. Acosta, N. Tajiri, J. Hoover, Y. Kaneko, and C. V. Borlongan, "Intravenous bone marrow stem cell grafts preferentially migrate to spleen and abrogate chronic inflammation in stroke," Stroke, vol. 46, no. 9, pp. 2616-2627, 2015.

[59] G. V. Goldmacher, R. Nasser, D. Y. Lee, S. Yigit, R. Rosenwasser, and L. Iacovitti, "Tracking transplanted bone marrow stem cells and their effects in the rat MCAO stroke model," PLOS ONE, vol. 8, no. 3, Article ID e60049, 2013.

[60] A. Vasconcelos-dos-Santos, P. H. Rosado-de-Castro, S. A. Lopes de Souza et al., "Intravenous and intra-arterial administration of bone marrow mononuclear cells after focal cerebral ischemia: is there a difference in biodistribution and efficacy?" Stem Cell Research, vol. 9, no. 1, pp. 1-8, 2012.

[61] C. Yang, L. Zhou, X. Gao et al., "Neuroprotective effects of bone marrow stem cells overexpressing glial cell line-derived neurotrophic factor on rats with intracerebral hemorrhage and neurons exposed to hypoxia/reoxygenation," Neurosurgery, vol. 68, no. 3, pp. 691-704, 2011.

[62] H. Liang, Y. Yin, T. Lin et al., "Transplantation of bone marrow stromal cells enhances nerve regeneration of the corticospinal tract and improves recovery of neurological functions in a collagenase-induced rat model of intracerebral hemorrhage," Molecules and Cells, vol. 36, no. 1, pp. 17-24, 2013.

[63] T. Menge, Y. Zhao, J. Zhao et al., "Traumatic brain injury: mesenchymal stem cells regulate blood-brain barrier integrity through TIMP3 release after traumatic brain injury," Science Translational Medicine, vol. 4, no. 161, Article ID 161ra150, 2012. 
[64] S. L. Gibb, Y. Zhao, D. Potter et al., "TIMP3 attenuates the loss of neural stem cells, mature neurons and neurocognitive dysfunction in traumatic brain injury," STEM CELLS, vol. 33, no. 12, pp. 3530-3544, 2015.

[65] M. R. Macleod, T. O'Collins, D. W. Howells, and G. A. Donnan, "Pooling of animal experimental data reveals influence of study design and publication bias," Stroke, vol. 35, no. 5, pp. 1203-1208, 2004.

[66] C. L. MacLellan, R. Paquette, and F. Colbourne, "A critical appraisal of experimental intracerebral hemorrhage research," Journal of Cerebral Blood Flow \& Metabolism, vol. 32, no. 4, pp. 612-627, 2012.

[67] M. A. Khalili, F. Sadeghian-Nodoushan, F. Fesahat, S. M. MirEsmaeili, M. Anvari, and S. H. Hekmati-moghadam, "Mesenchymal stem cells improved the ultrastructural morphology of cerebral tissues after subarachnoid hemorrhage in rats," Experimental Neurobiology, vol. 23, no. 1, pp. 77-85, 2014.

[68] J. A. Clayton and F. S. Collins, "Policy: NIH to balance sex in cell and animal studies," Nature, vol. 509, no. 7500, pp. 282-283, 2014.

[69] S. Gokhale, L. R. Caplan, and M. L. James, "Sex differences in incidence, pathophysiology, and outcome of primary intracerebral hemorrhage," Stroke, vol. 46, no. 3, pp. 886-892, 2015.

[70] B. Lei, B. Mace, S. T. Bellows et al., "Interaction between sex and apolipoprotein E genetic background in a murine model of intracerebral hemorrhage," Translational Stroke Research, vol. 3, no. 1, pp. 94-101, 2012.

[71] L.-L. Cui, E. Kerkelä, A. Bakreen et al., “The cerebral embolism evoked by intra-arterial delivery of allogeneic bone marrow mesenchymal stem cells in rats is related to cell dose and infusion velocity," Stem Cell Research and Therapy, vol. 6, article 11, 2015.

[72] M. Janowski, A. Lyczek, C. Engels et al., "Cell size and velocity of injection are major determinants of the safety of intracarotid stem cell transplantation," Journal of Cerebral Blood Flow and Metabolism, vol. 33, no. 6, pp. 921-927, 2013.

[73] V. Donega, C. H. Nijboer, C. T. J. van Velthoven et al., "Assessment of long-term safety and efficacy of intranasal mesenchymal stem cell treatment for neonatal brain injury in the mouse," Pediatric Research, vol. 78, no. 5, pp. 520-526, 2015.

[74] C. Suarez-Monteagudo, P. Hernandez-Ramirez, L. AlvarezGonzalez et al., "Autologous bone marrow stem cell neurotransplantation in stroke patients. An open study," Restorative Neurology and Neuroscience, vol. 27, no. 3, pp. 151-161, 2009.

[75] Z.-M. Li, Z.-T. Zhang, C.-J. Guo, F.-Y. Geng, F. Qiang, and L.$\mathrm{X}$. Wang, "Autologous bone marrow mononuclear cell implantation for intracerebral hemorrhage-a prospective clinical observation," Clinical Neurology and Neurosurgery, vol. 115, no. 1, pp. 72-76, 2013.

[76] A. Bhasin, M. V. Srivastava, S. S. Kumaran et al., "Autologous mesenchymal stem cells in chronic stroke," Cerebrovascular Diseases Extra, vol. 1, no. 1, pp. 93-104, 2011.

[77] A. Sharma, H. Sane, N. Gokulchandran et al., "Autologous bone marrow mononuclear cells intrathecal transplantation in chronic stroke," Stroke Research and Treatment, vol. 2014, Article ID 234095, 9 pages, 2014.

[78] J. Zhu, Y. Xiao, Z. Li et al., "Efficacy of surgery combined with autologous bone marrow stromal cell transplantation for treatment of intracerebral hemorrhage," Stem Cells International, vol. 2015, Article ID 318269, 7 pages, 2015.
[79] Administration UFaD, Guidance for industry: Preclinical assessment of investigational cellular and gene therapy products, 2013.

[80] B. Yang, X. Xi, J. Aronowski, and S. I. Savitz, "Ischemic stroke may activate bone marrow mononuclear cells to enhance recovery after stroke," Stem Cells and Development, vol. 21, no. 18, pp. 3332-3340, 2012.

[81] M. H. Askenase, S.-J. Han, A. L. Byrd et al., "Bone-marrowresident NK cells prime monocytes for regulatory function during infection," Immunity, vol. 42, no. 6, pp. 1130-1142, 2015.

[82] S. Knaän-Shanzer, "Concise review: the immune status of mesenchymal stem cells and its relevance for therapeutic application," Stem Cells, vol. 32, no. 3, pp. 603-608, 2014.

[83] S. Viswanathan and T. Bubela, "Current practices and reform proposals for the regulation of advanced medicinal products in Canada," Regenerative Medicine, vol. 10, no. 5, pp. 647-663, 2015.

[84] R. Mays and R. Deans, "Adult adherent cell therapy for ischemic stroke: clinical results and development experience using MultiStem," Transfusion, vol. 56, no. 4, pp. 6S-8S, 2016.

[85] P. M. George and G. K. Steinberg, "Novel stroke therapeutics: unraveling stroke pathophysiology and its impact on clinical treatments," Neuron, vol. 87, no. 2, pp. 297-309, 2015.

[86] F. Amiri, A. Jahanian-Najafabadi, and M. H. Roudkenar, "In vitro augmentation of mesenchymal stem cells viability in stressful microenvironments: in vitro augmentation of mesenchymal stem cells viability," Cell Stress and Chaperones, vol. 20, no. 2, pp. 237-251, 2015.

[87] L. Wei, J. L. Fraser, Z.-Y. Lu, X. Hu, and S. P. Yu, “Transplantation of hypoxia preconditioned bone marrow mesenchymal stem cells enhances angiogenesis and neurogenesis after cerebral ischemia in rats," Neurobiology of Disease, vol. 46, no. 3, pp. 635645, 2012.

[88] D. G. Phinney, "Functional heterogeneity of mesenchymal stem cells: implications for cell therapy," Journal of Cellular Biochemistry, vol. 113, no. 9, pp. 2806-2812, 2012.

[89] R. H. Lee, J. M. Yu, A. M. Foskett et al., "TSG-6 as a biomarker to predict efficacy of human mesenchymal stem/progenitor cells (hMSCs) in modulating sterile inflammation in vivo," Proceedings of the National Academy of Sciences of the United States of America, vol. 111, no. 47, pp. 16766-16771, 2014.

[90] C. A. Bravery, J. Carmen, T. Fong et al., "Potency assay development for cellular therapy products: an ISCT review of the requirements and experiences in the industry," Cytotherapy, vol. 15, no. 1, pp. 9-19, 2013.

[91] K. Kim, H. W. Park, H. E. Moon et al., "The effect of human umbilical cord blood-derived mesenchymal stem cells in a collagenase-induced intracerebral hemorrhage rat model," Experimental Neurobiology, vol. 24, no. 2, pp. 146-155, 2015.

[92] D. Yang, Y. Han, J. Zhang, A. Seyda, M. Chopp, and D. M. Seyfried, "Therapeutic effect of human umbilical tissuederived cell treatment in rats with experimental intracerebral hemorrhage," Brain Research, vol. 1444, pp. 1-10, 2012.

[93] M. Fatar, M. Stroick, M. Griebe et al., "Lipoaspirate-derived adult mesenchymal stem cells improve functional outcome during intracerebral hemorrhage by proliferation of endogenous progenitor cells: stem cells in intracerebral hemorrhages," Neuroscience Letters, vol. 443, no. 3, pp. 174-178, 2008.

[94] M. Strioga, S. Viswanathan, A. Darinskas, O. Slaby, and J. Michalek, "Same or not the same? comparison of adipose tissuederived versus bone marrow-derived mesenchymal stem and stromal cells," Stem Cells and Development, vol. 21, no. 14, pp. 2724-2752, 2012. 
[95] S. I. Savitz, M. Chopp, R. Deans, S. T. Carmichael, D. Phinney, and L. Wechsler, "Stem cell therapy as an emerging paradigm for stroke (STEPS) II," Stroke, vol. 42, no. 3, pp. 825-829, 2011.

[96] A. S. Arbab, B. Janic, J. Haller, E. Pawelczyk, W. Liu, and J. A. Frank, "In vivo cellular imaging for translational medical research," Current Medical Imaging Reviews, vol. 5, no. 1, pp. 1938, 2009.

[97] K. C. Nam, Y. J. Yong, J.-S. Park et al., "Tracking of neural stem cells in rats with intracerebral hemorrhage by the use of 3T MRI," Korean Journal of Radiology, vol. 9, no. 3, pp. 196-204, 2008.

[98] E. Farrell, P. Wielopolski, P. Pavljasevic et al., "Effects of iron oxide incorporation for long term cell tracking on MSC differentiation in vitro and in vivo," Biochemical and Biophysical Research Communications, vol. 369, no. 4, pp. 1076-1081, 2008.

[99] H. S. Kim, S. Y. Oh, H. J. Joo, K.-R. Son, I.-C. Song, and W. K. Moon, "The effects of clinically used MRI contrast agents on the biological properties of human mesenchymal stem cells," NMR in Biomedicine, vol. 23, no. 5, pp. 514-522, 2010.

[100] Jasmin, A. L. M. Torres, L. Jelicks, A. C. C. De Carvalho, D. C. Spray, and R. Mendez-Otero, "Labeling stem cells with superparamagnetic iron oxide nanoparticles: analysis of the labeling efficacy by microscopy and magnetic resonance imaging," Methods in Molecular Biology, vol. 906, pp. 239-252, 2012.

[101] Jasmin, A. L. M. Torres, H. M. P. Nunes et al., "Optimized labeling of bone marrow mesenchymal cells with superparamagnetic iron oxide nanoparticles and in vivo visualization by magnetic resonance imaging," Journal of Nanobiotechnology, vol. 9, article 4, 2011.

[102] T. Singhal, "Positron emission tomography applications in clinical neurology," Seminars in Neurology, vol. 32, no. 4, pp. 421-431, 2012.

[103] V. Battistella, G. R. de Freitas, L. M. B. da Fonseca et al., "Safety of autologous bone marrow mononuclear cell transplantation in patients with nonacute ischemic stroke," Regenerative Medicine, vol. 6, no. 1, pp. 45-52, 2011.

[104] P. H. Rosado-de-Castro, F. R. Schmidt, V. Battistella et al., "Biodistribution of bone marrow mononuclear cells after intraarterial or intravenous transplantation in subacute stroke patients," Regenerative Medicine, vol. 8, no. 2, pp. 145-155, 2013.

[105] L. M. Barbosa da Fonseca, V. Battistella, G. R. de Freitas et al., "Early tissue distribution of bone marrow mononuclear cells after intra-arterial delivery in a patient with chronic stroke," Circulation, vol. 120, no. 6, pp. 539-541, 2009.

[106] L. M. Barbosa da Fonseca, B. Gutfilen, P. H. Rosado de Castro et al., "Migration and homing of bone-marrow mononuclear cells in chronic ischemic stroke after intra-arterial injection," Experimental Neurology, vol. 221, no. 1, pp. 122-128, 2010.

[107] P. L. Correa, C. T. Mesquita, R. M. Felix et al., "Assessment of intra-arterial injected autologous bone marrow mononuclear cell distribution by radioactive labeling in acute ischemic stroke," Clinical Nuclear Medicine, vol. 32, no. 11, pp. 839-841, 2007.

[108] O. Detante, A. Moisan, J. Dimastromatteo et al., "Intravenous administration of 99mTc-HMPAO-labeled human mesenchymal stem cells after stroke: in vivo imaging and biodistribution," Cell Transplantation, vol. 18, no. 12, pp. 1369-1379, 2009. 

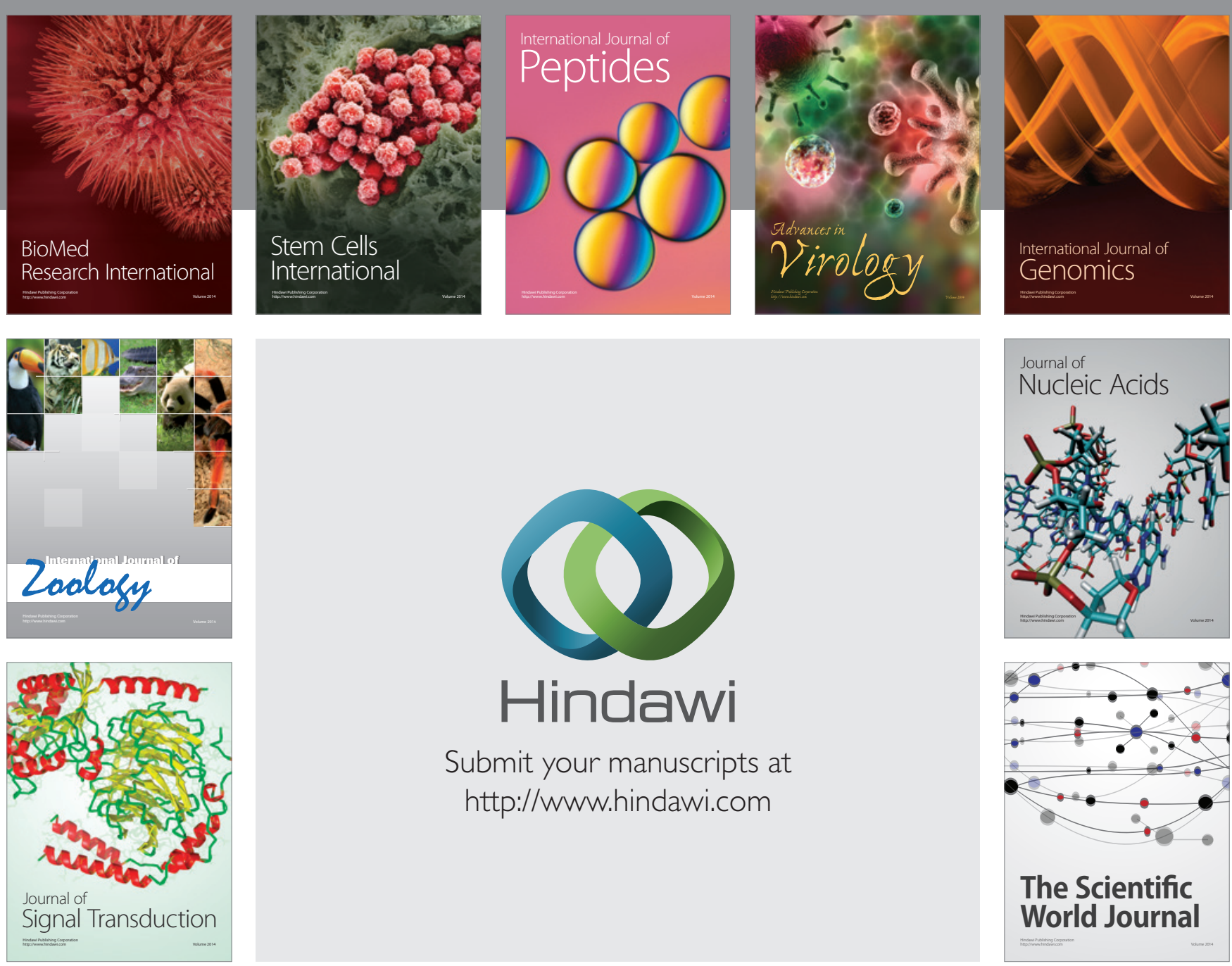

Submit your manuscripts at

http://www.hindawi.com
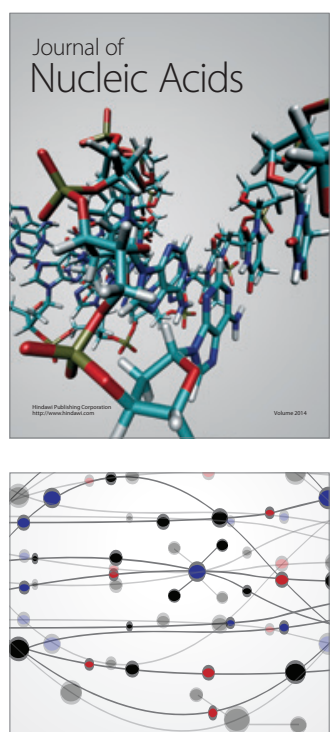

The Scientific World Journal
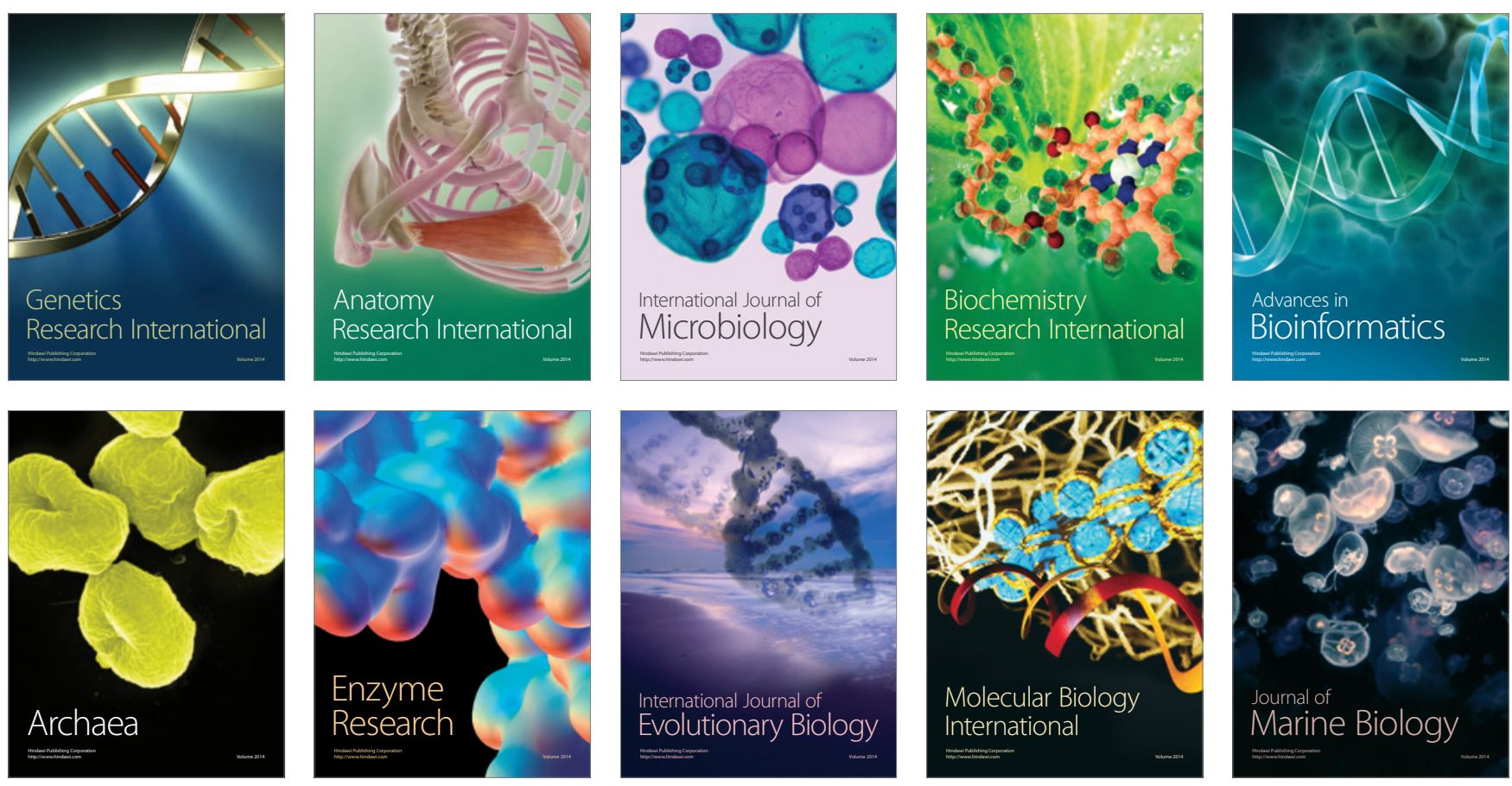